\title{
Investigation of the $p$-Laplacian nonperiodic nonlinear boundary value problem via generalized Caputo fractional derivatives
}

\author{
M.M. Matar' ${ }^{1}$ M.I. Abbas², J. Alzabut ${ }^{3}$, M.K.A. Kaabar ${ }^{4}$, S. Etemad ${ }^{5}$ and S. Rezapour ${ }^{6,7,8^{*}}$ (1)
}

\section{"Correspondence:}

shahramrezapour@duytan.edu.vn;

sh.rezapour@mail.cmuh.org.tw;

sh.rezapour@azaruniv.ac.ir;

rezapourshahram@yahoo.ca

${ }^{6}$ Institute of Research and

Development, Duy Tan University, Da Nang 550000, Vietnam

${ }^{7}$ Faculty of Natural Sciences, Duy Tan University, Da Nang 550000,

Vietnam

Full list of author information is available at the end of the article

\begin{abstract}
A newly proposed $p$-Laplacian nonperiodic boundary value problem is studied in this research paper in the form of generalized Caputo fractional derivatives. The existence and uniqueness of solutions are fully investigated for this problem using some fixed point theorems such as Banach and Schauder. This work is supported with an example to apply all obtained new results and validate their applicability.
\end{abstract}

MSC: Primary 34A08; secondary 34A12

Keywords: $p$-Laplacian; Fractional-order differential system; Banach and Schauder fixed point results; The generalized Caputo fractional derivative

\section{Introduction}

The fractional calculus theory has recently become an interesting topic of research for mathematicians, scientists, and engineers due to the appearance of this theory in several applications in natural science and engineering and the ability to model many systems and phenomena that have memory effects. For more resources about this theory, we suggest [1-12]. Several new fractional derivatives' definitions have been proposed in the last decade, and the most common ones are Caputo, Riemann-Liouville, and Hadamard fractional derivatives. Therefore, fractional-order differential equations can be formulated in the form of various fractional differential operators. As a result, several generalized formulations of fractional differential operators have been introduced to effectively combine other operators to avoid the confusion when working with various existing fractional differential operators $[13,14]$.

Studying the fractional boundary value problems (FcBVPrs) has attracted a special interest of many mathematicians due to the various applications of fractional differential equations in several engineering and scientific fields. Some recent research studies have been conducted on proving the existence and uniqueness of FcBVPrs [15-35]. While there are some studies that have applied various methods depending on the proposed fractional formulation and the selected initial/boundary conditions, some other studies have discussed the FcBVPrs involving $p$-Laplacian operator such as the investigation of the turbulent flow in a porous medium (refer to [36-41]).

(c) The Author(s) 2021. This article is licensed under a Creative Commons Attribution 4.0 International License, which permits use sharing, adaptation, distribution and reproduction in any medium or format, as long as you give appropriate credit to the original author(s) and the source, provide a link to the Creative Commons licence, and indicate if changes were made. The images or other third party material in this article are included in the article's Creative Commons licence, unless indicated otherwise in a credit line to the material. If material is not included in the article's Creative Commons licence and your intended use is not permitted by statutory regulation or exceeds the permitted use, you will need to obtain permission directly from the copyright holder. To view a copy of this licence, visit http://creativecommons.org/licenses/by/4.0/. 
Both Katugampola and Almeida defined and developed new nonlocal notions of fractional derivatives, known as the generalized Caputo fractional (GCpFr) derivative (see $[42,43])$. One of the main advantages of the GCpFr derivative is the ability to combine all traditional fractional derivatives, and it satisfies the semigroup property, hence, $\mathrm{GCpFr}$ derivative is considered a generalized form of fractional derivatives. There are a number of research studies that have been done in the form of GCpFr derivative [43-45]. However, according to the authors' knowledge, there are no existing research studies on $\mathrm{GCpFr}$ involving $p$-Laplacian operator.

The authors in [40] proved the solutions' existence for the following fractional differential equation:

$$
\mathfrak{D}^{\beta} \varphi_{p}\left(\mathfrak{D}^{\alpha} u(t)\right)=q\left(t, u(t), \mathfrak{D}^{\gamma} u(t)\right), \quad 0 \leq t \leq 1,
$$

subject to the following $p$-Laplacian operator and integral boundary conditions:

$$
\left\{\begin{array}{l}
u(0)+\epsilon_{1} u(1)=\lambda_{1} \int_{0}^{1} g(s, u(s)) d s, \\
u^{\prime}(0)+\epsilon_{2} u^{\prime}(1)=\lambda_{2} \int_{0}^{1} h(s, u(s)) d s, \\
\mathfrak{D}^{\alpha} x(0)=0, \quad \mathfrak{D}^{\alpha} u(1)=\zeta \mathfrak{D}^{\alpha} u(\zeta),
\end{array}\right.
$$

where $q, g$, and $h$ are continuous functions. $\mathfrak{D}^{\alpha}, \mathfrak{D}^{\beta}$ are the Caputo fractional derivatives of orders $\alpha, \beta$ such that $\alpha, \beta \in(1,2] . \zeta, \epsilon_{j}, \lambda_{j}(j=1,2)$ are nonnegative parameters.

The following is the integral boundary value problem involving a fractional $p$-Laplacian equation in the form of mixed fractional derivatives [39]:

$$
\left\{\begin{array}{l}
\mathfrak{D}_{0^{+}}^{\beta} \varphi_{p}\left(\mathfrak{D}_{0^{+}}^{\alpha} u(t)\right)=q\left(t, u(t), \mathfrak{D}^{\gamma} u(t)\right), \quad 0 \leq t \leq 1, \\
u(0)=\epsilon \int_{0}^{1} u(s) d s+v u(\xi), \\
\mathfrak{D}_{0^{+}}^{\alpha} u(0)=j \mathfrak{D}_{0^{+}}^{\alpha} u(\zeta),
\end{array}\right.
$$

where $q \in C\left([0,1] \times \mathbb{R}^{2}, \mathbb{R}\right)$ is a nonlinear function, $\mathfrak{D}_{0^{+}}^{\beta}$ and $\mathfrak{D}_{0^{+}}^{\alpha}$ are the Caputo fractional (CapFr) derivatives of orders $\alpha, \beta$ such that $\alpha, \beta \in(0,1], \varphi_{p}$ is the $p$-Laplacian operator, $p>1, \epsilon, v, j \in \mathbb{R}, \xi, \zeta \in[0,1]$.

We study the following fractional differential equation as we got motivated by the above studies:

$$
\left\{\begin{array}{lr}
\frac{d}{d t}\left(\varphi_{p}\left({ }^{C} \mathfrak{D}^{\alpha, \rho} u(t)\right)\right)=q\left(t, u(t),{ }^{C} \mathfrak{D}^{\gamma, \rho} u(t)\right) & (0 \leq t \leq 1), \\
u(0)+\mu u(1)=\theta(u(0), u(1)), & u^{\prime}(1)=v(u(0), u(1)),
\end{array}\right.
$$

where ${ }^{C} \mathfrak{D}^{\alpha, \rho}$ and ${ }^{C} \mathfrak{D}^{\gamma, \rho} u(t), \alpha \in(1,2), \gamma \in(0,1), \rho>1$, are GCpFr derivatives, $\varphi_{p}, p>1$ is a $p$-Laplacian operator, $\mu \neq-1$, and the nonlinear functions $q:[0,1] \times \mathbb{R} \times \mathbb{R} \rightarrow \mathbb{R}$ and $\theta, v: \mathbb{R} \times \mathbb{R} \rightarrow \mathbb{R}$ are given continuous functions. The main goal of this research paper is to prove the existence and uniqueness of equation (3) solutions by applying the Banach and Schauder fixed point theorems. Then, we apply our results by introducing an applicable example to validate all our obtained results. One may observe that the results of this paper are presented in a form which is essentially of high degree of generality. For particular values of $\rho$, equation (3) can be reduced to (1) and (2). One can find mathematical models 
of different physical phenomena in the world as simple cases of equation (3), such as blowup. One can find distinct applications of mathematical models in the literature (see, for example, [46-48]).

\section{Basic preliminaries}

The following is a review of some important definitions and properties in the theory of fractional differential equations involving the GCpFr derivatives. Note that we have adapted some notations from $[3,49]$.

Definition 1 (Generalized Caputo fractional integral (GCpFrI)) Assume that $\alpha \in(m-$ $1, m), m \in \mathbb{N}$, and $\rho>0$. The GCpFrI of order $\alpha$ for a given function $q:[0,1] \rightarrow \mathbb{R}$ with respect to parameter $\rho$ can be written in the following form:

$$
\mathcal{I}^{\alpha, \rho} q(t)=\frac{\rho^{1-\alpha}}{\Gamma(\alpha)} \int_{0}^{t}\left(t^{\rho}-s^{\rho}\right)^{\alpha-1} s^{\rho-1} q(s) d s,
$$

provided that the integral exists.

The GCpFrI is linear and satisfies the following semigroup/ index property:

(I) $\mathcal{I}^{\alpha, \rho}\left(c_{1} q(t)+c_{2} g(t)\right)=c_{1} \mathcal{I}^{\alpha, \rho} q(t)+c_{2} \mathcal{I}^{\alpha, \rho} g(t), c_{1}, c_{2} \in \mathbb{R}$,

(II) $\mathcal{I}^{\alpha, \rho}\left(\mathcal{I}^{\beta, \rho} q(t)\right)=\mathcal{I}^{\alpha+\beta, \rho} q(t), \alpha, \beta>0$.

In addition, if we assume the existence of the first derivative of $\mathcal{I}^{\alpha, \rho} f(t)$, we have the following:

$$
\frac{d}{d t} \mathcal{I}^{\alpha, \rho} q(t)=t^{\rho-1} \mathcal{I}^{\alpha-1, \rho} q(t)
$$

Definition 2 Assume that $q:[0,1] \rightarrow \mathbb{R}$, where $q \in C^{m}[0,1]$. The GCpFr derivative of order $\alpha \in(m-1, m)$ is written as follows:

$$
\begin{aligned}
C_{\mathfrak{D}^{\alpha, \rho}}^{\alpha}(t) & =\mathcal{I}^{m-\alpha, \rho}\left(\left(s^{1-\rho} q\right)^{(m)}\right)(t) \\
& =\frac{\rho^{1-m+\alpha}}{\Gamma(m-\alpha)} \int_{0}^{t}\left(t^{\rho}-s^{\rho}\right)^{m-\alpha-1}\left(s^{1-\rho} q\right)^{(m)}(s) d s .
\end{aligned}
$$

Remark 1 Both of the GCpFr derivative and GCpFrI formulated in (4) and (6) generalize the traditional fractional derivative. GCpFr derivative and GCpFrI are forms of the Caputo-Katugampola fractional derivative and integral, respectively. For example, if we assume $\rho=1$, then the GCpFr derivative and GCpFrI can be reduced to the CapFr derivative and Riemann-Liouville fractional (RIFr) integral, respectively, as follows:

$$
{ }^{C} \mathfrak{D}^{\alpha} q(t):={ }^{C} \mathfrak{D}^{\alpha, 1} q(t)=\mathcal{I}^{m-\alpha} q^{(m)}(t)=\frac{1}{\Gamma(m-\alpha)} \int_{0}^{t}(t-s)^{m-\alpha-1} q^{(m)}(s) d s
$$

and

$$
\mathcal{I}^{\alpha} q(t):=\mathcal{I}^{\alpha, 1} q(t)=\frac{1}{\Gamma(\alpha)} \int_{0}^{t}(t-s)^{\alpha-1} q(s) d s, \quad \alpha>0 .
$$

On the contrary, if we let $\rho \rightarrow 0^{+}$, the GCpFr derivative and GCpFrI can be reduced to the Caputo-Hadamard derivative and Hadamard fractional integral, respectively, as 
follows:

$$
{ }^{H} \mathfrak{D}^{\alpha} q(t)={ }^{H} \mathcal{I}^{m-\alpha} q^{(m)}(t)
$$

and

$$
{ }^{H} \mathcal{I}^{\alpha} q(t)=\frac{1}{\Gamma(\alpha)} \int_{0}^{t}\left(\ln \frac{t}{s}\right)^{\alpha-1} \frac{q(s)}{s} d s
$$

As a result, we can keep the traditional forms of fractional derivatives by selecting particular parameters for the GCpFr derivative.

Lemma 3 The following relations are valid:

(i) Let $0<\gamma<\alpha, q \in C[0,1]$, then

$$
{ }^{C} \mathfrak{D}^{\gamma, \rho} \mathcal{I}^{\alpha, \rho} q(t)=\mathcal{I}^{\alpha-\gamma, \rho} q(t)
$$

(ii) Let $0<\alpha<\beta$, then

$$
C_{\mathfrak{D}^{\alpha, \rho}}\left(\frac{t^{\rho}}{\rho}\right)^{\beta-1}=\frac{\Gamma(\beta)}{\Gamma(\beta-\alpha)}\left(\frac{t^{\rho}}{\rho}\right)^{\beta-\alpha-1} .
$$

In Figs. 1 and 2, the dynamical behavior of the GCpFr derivative can be observed on four given functions $u(t)=\frac{t^{4}}{4}, u(t)=\frac{t^{6}}{8}, u(t)=\frac{t^{6}}{9}$, and $u(t)=\frac{t^{9}}{27}$ for $\rho=2$ and $\rho=3$, respectively.

We discuss the following result due to its importance in finding a solution for the fractional differential equation formulated in the form of GCpFr derivative.

Lemma 4 Assume that $\alpha \in(m-1, m), \rho>0$, and $f \in C^{m}[0,1]$. Then

$$
\mathcal{I}^{\alpha, \rho C} \mathfrak{D}^{\alpha, \rho} q(t)=q(t)+c_{0}+c_{1}\left(\frac{t^{\rho}}{\rho}\right)+c_{2}\left(\frac{t^{\rho}}{\rho}\right)^{2}+\cdots+c_{m-1}\left(\frac{t^{\rho}}{\rho}\right)^{m-1}
$$

and ${ }^{C} \mathfrak{D}^{\alpha, \rho} \mathcal{I}^{\alpha, \rho} q(t)=q(t)$ for $c_{k} \in \mathbb{R}$ and $k=0,1, \ldots, m-1$.

The following is a review of some important properties of the $p$-Laplacian operator that will be applied in this research paper in the next sections. For more information about those properties, we refer to [38, 39].

Definition 5 The following $p$-Laplacian operator is formulated:

$$
\varphi_{p}(u)=|u|^{p-2} u=u^{p-1}, \quad u \geq 0, p>1,
$$

such that $\varphi_{p}{ }^{-1}=\varphi_{r}$, where $\frac{1}{p}+\frac{1}{r}=1$. 

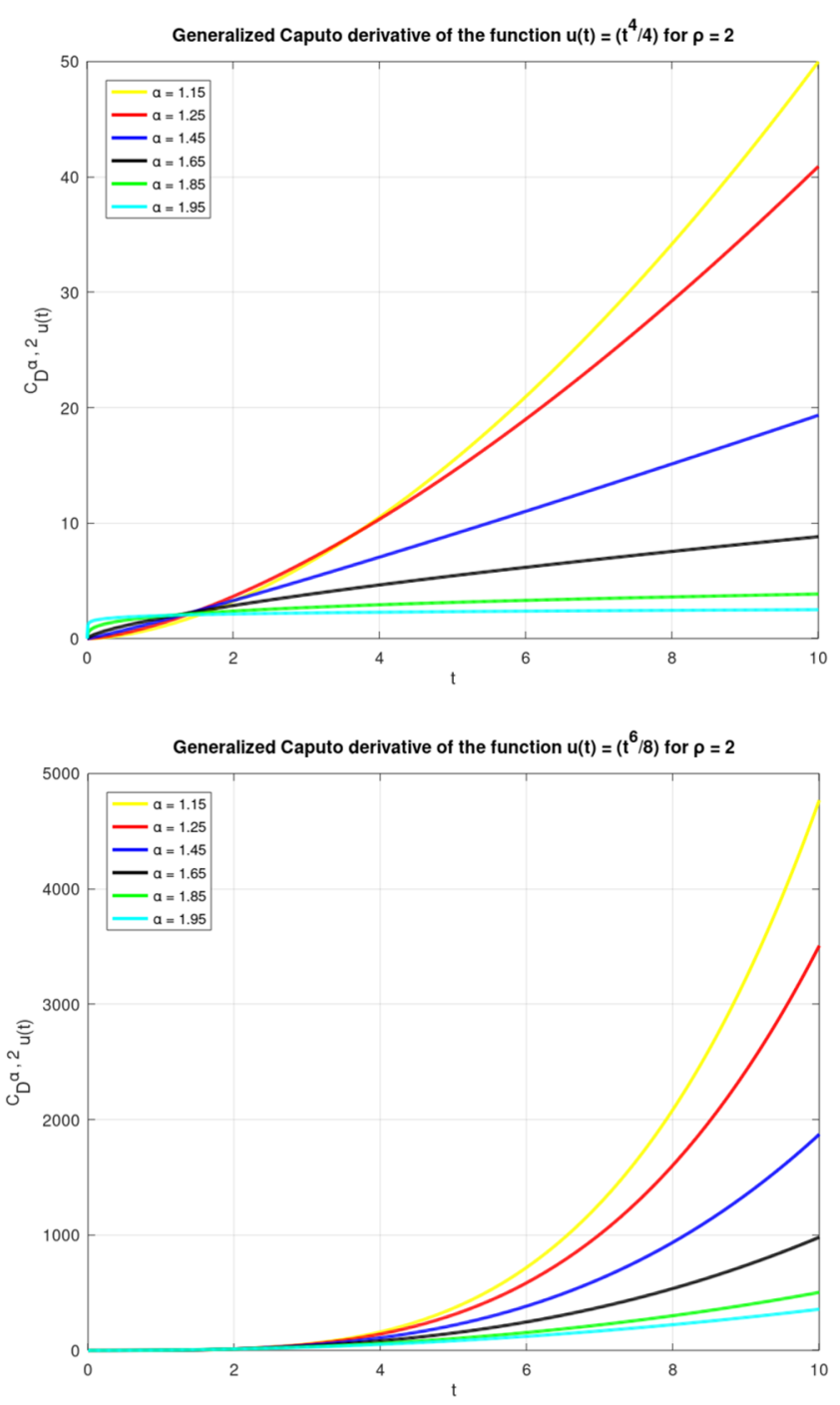

Figure 1 The GCpFr derivative of $u(t)=\frac{t^{4}}{4}, \frac{t^{6}}{8}$ for $\rho=2$

Lemma 6 Assume that $\varphi_{p}(u), p \geq 2$, is a $p$-Laplacian operator and $|u|,|m| \leq M$. Then we have the following:

$$
\left|\varphi_{p}(u)-\varphi_{p}(m)\right| \leq(p-1) M^{p-2}|u-m| .
$$

Our results in this research paper will be based on applying the following Banach and Schauder fixed point theorems.

Theorem 7 (Banach's fixed point [50]) Assume that $\mathbb{T}^{*}$ is a contraction mapping from a closed subset $\mathcal{K}$ of a Banach space $\mathcal{X}$ into itself. Then there exists unique $z \in \mathcal{K}$ such that $\mathbb{T}^{*}(z)=z$.

Theorem 8 (Schauder's fixed point [50]) Assume that $\mathbb{D}$ is a nonempty closed convex subset of a Banach space $\mathcal{U}$.If $\mathbb{T}^{*}: \mathbb{D} \rightarrow \mathbb{D}$ is a compact operator, then $\mathbb{T}^{*}$ has at least one fixed point in $\mathbb{D}$. 

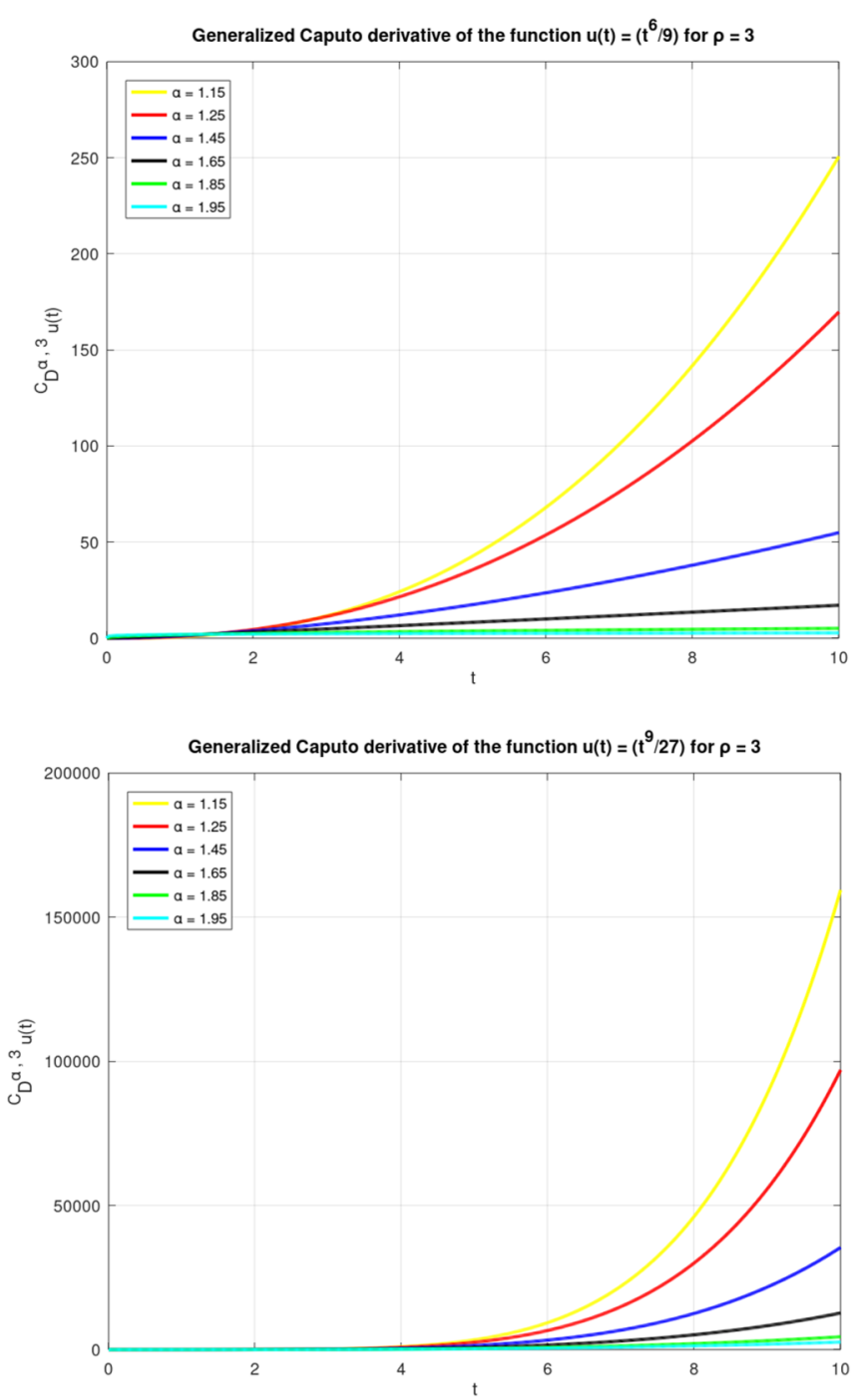

Figure 2 The GCpFr derivative of $u(t)=\frac{t^{6}}{9}, \frac{t^{9}}{27}$ for $\rho=3$

\section{Main results}

By using the fixed point theorems, we study here the existence and uniqueness of solutions for system (3). Let us consider the following linear equation:

$$
\left\{\begin{array}{l}
\frac{d}{d t}\left(\varphi_{p}\left({ }^{C} \mathfrak{D}^{\alpha, \rho} u(t)\right)\right)=w(t) \quad(t \in[0,1]), \\
u(0)+\mu u(1)=\theta, \quad u^{\prime}(1)=v
\end{array}\right.
$$

Lemma 9 ([29]) Suppose that $w \in C[0,1], \alpha \in(1,2), \rho>1$, and $\mu \neq-1$. Then the boundary value problem (BoVaPr) (9) has a solution expressed as follows:

$$
\begin{aligned}
u(t)= & \frac{\theta}{\mu+1}-\frac{\mu \nu}{\rho(\mu+1)}+\frac{v}{\rho} t^{\rho} \\
& +\frac{\rho^{1-\alpha}}{\Gamma(\alpha)} \int_{0}^{t}\left(t^{\rho}-\tau^{\rho}\right)^{\alpha-1} \tau^{\rho-1} \varphi_{r}\left(\int_{0}^{\tau} w(s) d s\right) d \tau
\end{aligned}
$$




$$
\begin{aligned}
& -\frac{\rho^{1-\alpha} t^{\rho}}{\Gamma(\alpha-1)} \int_{0}^{1}\left(1-\tau^{\rho}\right)^{\alpha-2} \tau^{\rho-1} \varphi_{r}\left(\int_{0}^{\tau} w(s) d s\right) d \tau \\
& -\frac{\mu \rho^{1-\alpha}}{(\mu+1) \Gamma(\alpha)} \int_{0}^{1}\left(1-\tau^{\rho}\right)^{\alpha-1} \tau^{\rho-1} \varphi_{r}\left(\int_{0}^{\tau} w(s) d s\right) d \tau \\
& +\frac{\mu \rho^{1-\alpha}}{(\mu+1) \Gamma(\alpha-1)} \int_{0}^{1}\left(1-\tau^{\rho}\right)^{\alpha-2} \tau^{\rho-1} \varphi_{r}\left(\int_{0}^{\tau} w(s) d s\right) d \tau .
\end{aligned}
$$

Proof By integrating the fractional differential equation in (9), we obtain

$$
\varphi_{p}\left({ }^{C} \mathfrak{D}^{\alpha, \rho} u(t)\right)=\varphi_{p}\left({ }^{C} \mathfrak{D}^{\alpha, \rho} u(0)\right)+\int_{0}^{t} w(s) d s .
$$

The definition of generalized Caputo derivative implies that ${ }^{C} \mathfrak{D}^{\alpha, \rho} u(0)=0$, and by using the properties of Laplacian operators, we have

$$
{ }^{C} \mathfrak{D}^{\alpha, \rho} u(t)=\varphi_{r}\left(\int_{0}^{t} w(s) d s\right) .
$$

By applying the generalized Caputo integral $\mathcal{I}^{\alpha, \rho}$ to both sides, we deduce the following:

$$
u(t)=\mathcal{I}^{\alpha, \rho} \varphi_{r}\left(\int_{0}^{t} w(s) d s\right)+a_{0}+a_{1} t^{\rho}
$$

for some constants $a_{0}$ and $a_{1}$. Accordingly, by using (5), we obtain

$$
u^{\prime}(t)=\frac{\rho^{2-\alpha}}{\Gamma(\alpha-1)} \int_{0}^{t}\left(t^{\rho}-\tau^{\rho}\right)^{\alpha-2}(t \tau)^{\rho-1} \varphi_{r}\left(\int_{0}^{\tau} w(s) d s\right) d \tau+a_{1} \rho t^{\rho-1} .
$$

Then we have

$$
u(1)=\frac{\rho^{1-\alpha}}{\Gamma(\alpha)} \int_{0}^{1}\left(1-\tau^{\rho}\right)^{\alpha-1} \tau^{\rho-1} \varphi_{r}\left(\int_{0}^{\tau} w(s) d s\right) d \tau+a_{0}+a_{1}
$$

and

$$
u^{\prime}(1)=\frac{\rho^{2-\alpha}}{\Gamma(\alpha-1)} \int_{0}^{1}\left(1-\tau^{\rho}\right)^{\alpha-2} \tau^{\rho-1} \varphi_{r}\left(\int_{0}^{\tau} w(s) d s\right) d \tau+a_{1} \rho .
$$

It is obvious that $u(0)=a_{0}$. Hence, by the boundary conditions, we deduce the following:

$$
a_{1}=\frac{\nu}{\rho}-\frac{\rho^{1-\alpha}}{\Gamma(\alpha-1)} \int_{0}^{1}\left(1-\tau^{\rho}\right)^{\alpha-2} \tau^{\rho-1} \varphi_{r}\left(\int_{0}^{\tau} w(s) d s\right) d \tau
$$

and

$$
\begin{aligned}
a_{0}= & \frac{\theta}{\mu+1}-\frac{\mu \nu}{\rho(\mu+1)} \\
& -\frac{\mu \rho^{1-\alpha}}{(\mu+1) \Gamma(\alpha)} \int_{0}^{1}\left(1-\tau^{\rho}\right)^{\alpha-1} \tau^{\rho-1} \varphi_{r}\left(\int_{0}^{\tau} h(s) d s\right) d \tau
\end{aligned}
$$




$$
+\frac{\mu \rho^{1-\alpha}}{(\mu+1) \Gamma(\alpha-1)} \int_{0}^{1}\left(1-\tau^{\rho}\right)^{\alpha-2} \tau^{\rho-1} \varphi_{r}\left(\int_{0}^{\tau} h(s) d s\right) d \tau
$$

Substituting $a_{0}$ and $a_{1}$ in (11), we get solution (10). This completes the proof.

Assume that $\mathfrak{C}:=C([0,1], \mathbb{R})$ is the Banach space of all continuous real-valued functions defined on $[0,1]$ endowed with the usual supremum norm defined by $\|u\|=\sup _{t \in[0,1]}|u(t)|$.

Let $\mathbf{U}=\left\{u: u \in \mathfrak{C},{ }^{C} \mathfrak{D}^{\gamma, \rho} u \in \mathfrak{C}\right\}$, then $\mathbf{U}$ is a Banach space endowed with the norm

$$
\|u\|_{\mathbf{U}}=\|u\|+\left\|{ }^{C} \mathfrak{D}^{\gamma, \rho} u\right\|
$$

By virtue of Lemma 9, we deduce that the solution of equation (3) is expressed as follows:

$$
\begin{aligned}
u(t)= & \frac{\theta(u(0), u(1))}{\mu+1}-\frac{\mu \nu(u(0), u(1))}{\rho(\mu+1)}+\frac{v(u(0), u(1))}{\rho} t^{\rho} \\
& +\frac{\rho^{1-\alpha}}{\Gamma(\alpha)} \int_{0}^{t}\left(t^{\rho}-\tau^{\rho}\right)^{\alpha-1} \tau^{\rho-1} \varphi_{r}\left(\int_{0}^{\tau} q\left(s, u(s),{ }^{C} \mathfrak{D}^{\gamma, \rho} u(s)\right) d s\right) d \tau \\
& -\frac{\rho^{2-\alpha} t^{\rho}}{\Gamma(\alpha-1)} \int_{0}^{1}\left(1-\tau^{\rho}\right)^{\alpha-2} \tau^{\rho-1} \varphi_{r}\left(\int_{0}^{\tau} q\left(s, u(s),{ }^{C} \mathfrak{D}^{\gamma, \rho} u(s)\right) d s\right) d \tau \\
& -\frac{\mu \rho^{1-\alpha}}{(\mu+1) \Gamma(\alpha)} \int_{0}^{1}\left(1-\tau^{\rho}\right)^{\alpha-1} \tau^{\rho-1} \varphi_{r}\left(\int_{0}^{\tau} q\left(s, u(s),{ }^{C} \mathfrak{D}^{\gamma, \rho} u(s)\right) d s\right) d \tau \\
& +\frac{\mu \rho^{2-\alpha}}{(\mu+1) \Gamma(\alpha-1)} \int_{0}^{1}\left(1-\tau^{\rho}\right)^{\alpha-2} \tau^{\rho-1} \varphi_{r}\left(\int_{0}^{\tau} q\left(s, u(s),{ }^{C} \mathfrak{D}^{\gamma, \rho} u(s)\right) d s\right) d \tau .
\end{aligned}
$$

Let us now pose the following conditions to prove our next result:

(H1) The function $q:[0,1] \times \mathbb{R} \times \mathbb{R} \rightarrow \mathbb{R}$ is jointly continuous, and there exists a constant $L_{q}>0$ such that, for $u_{i}, m_{i} \in \mathbb{R}(i=1,2)$,

$$
\left|q\left(t, u_{1}, m_{1}\right)-q\left(t, u_{2}, m_{2}\right)\right| \leq L_{q}\left(\left|u_{1}-u_{2}\right|+\left|m_{1}-m_{2}\right|\right) \quad(t \in[0,1]) .
$$

(H2) The functionals $\theta, v: \mathbb{R} \times \mathbb{R} \rightarrow \mathbb{R}$ have the respective upper bounds $M_{\theta}$ and $M_{v}$.

(H3) There exists a finite solution $l>0$ of the following inequality:

$$
\begin{aligned}
l \geq & \frac{M_{\theta}}{|\mu+1|}+\frac{|\mu| M_{v}}{\rho|\mu+1|}+\frac{M_{v}}{\rho}+\frac{M_{v}}{\rho^{1-\gamma} \Gamma(2-\gamma)} \\
& +\frac{(\alpha \rho+1)(2|\mu|+1) M^{r-1}}{\rho^{\alpha}|\mu+1| \Gamma(\alpha+1)}+\frac{M^{r-1}}{\rho^{\alpha-\gamma} \Gamma(\alpha-\gamma+1)}+\frac{M^{r-1}}{\rho^{\alpha-\gamma-1} \Gamma(2-\gamma) \Gamma(\alpha)},
\end{aligned}
$$

where $M:=L_{q} l+q^{*}$ and $q^{*}:=\sup _{t \in[0,1]}|q(t, 0,0)|$.

For the sake of abbreviation, we set

$$
\begin{aligned}
& \Lambda_{1}:=\frac{(r-1) M^{r-2} L_{q}(2|\mu|+1)(1+(\alpha \rho+1)) \mathbf{B}\left(\alpha, \frac{1}{\rho}+1\right)}{\rho^{\alpha}|\mu+1| \Gamma(\alpha)}, \\
& \Lambda_{2}:=\frac{(r-1) M^{r-2} L_{q}}{\rho^{\alpha-\gamma}}\left[\frac{\mathbf{B}\left(\alpha-\gamma, \frac{1}{\rho}+1\right)}{\Gamma(\alpha-\gamma)}+\frac{\rho \mathbf{B}\left(\alpha-1, \frac{1}{\rho}+1\right)}{\Gamma(2-\gamma) \Gamma(\alpha-1)}\right],
\end{aligned}
$$


where $\mathbf{B}(\cdot, \cdot)$ denotes the well-known beta function defined by

$$
\mathbf{B}(m, n)=\frac{\Gamma(m) \Gamma(n)}{\Gamma(m+n)}=\int_{0}^{1}(1-s)^{m-1} s^{n-1} d s \quad(m>0, n>0) .
$$

An operator, denoted by $\Psi: \mathbf{U} \rightarrow \mathbf{U}$, can be defined as follows:

$$
\begin{aligned}
\Psi u(t)= & \frac{\theta(u(0), u(1))}{\mu+1}-\frac{\mu v(u(0), u(1))}{\rho(\mu+1)}+\frac{v(u(0), u(1))}{\rho} t^{\rho} \\
& +\frac{\rho^{1-\alpha}}{\Gamma(\alpha)} \int_{0}^{t}\left(t^{\rho}-\tau^{\rho}\right)^{\alpha-1} \tau^{\rho-1} \varphi_{r}\left(\int_{0}^{\tau} q\left(s, u(s),{ }^{C} \mathfrak{D}^{\gamma, \rho} u(s)\right) d s\right) d \tau \\
& -\frac{\rho^{2-\alpha} t^{\rho}}{\Gamma(\alpha-1)} \int_{0}^{1}\left(1-\tau^{\rho}\right)^{\alpha-2} \tau^{\rho-1} \varphi_{r}\left(\int_{0}^{\tau} q\left(s, u(s),{ }^{C} \mathfrak{D}^{\gamma, \rho} u(s)\right) d s\right) d \tau \\
& -\frac{\mu \rho^{1-\alpha}}{(\mu+1) \Gamma(\alpha)} \int_{0}^{1}\left(1-\tau^{\rho}\right)^{\alpha-1} \tau^{\rho-1} \varphi_{r}\left(\int_{0}^{\tau} q\left(s, u(s),{ }^{C} \mathfrak{D}^{\gamma, \rho} u(s)\right) d s\right) d \tau \\
& +\frac{\mu \rho^{2-\alpha}}{(\mu+1) \Gamma(\alpha-1)} \int_{0}^{1}\left(1-\tau^{\rho}\right)^{\alpha-2} \tau^{\rho-1} \varphi_{r}\left(\int_{0}^{\tau} q\left(s, u(s),{ }^{C} \mathfrak{D}^{\gamma, \rho} u(s)\right) d s\right) d \tau .
\end{aligned}
$$

By virtue of Lemma 3, we deduce

$$
\begin{aligned}
{ }^{C} \mathfrak{D}^{\gamma, \rho} \Psi u(t) \\
=\frac{v(u(0), u(1))}{\rho^{1-\gamma} \Gamma(2-\gamma)} t^{\rho(1-\gamma)} \\
\quad+\frac{\rho^{1-\alpha+\gamma}}{\Gamma(\alpha-\gamma)} \int_{0}^{t}\left(t^{\rho}-\tau^{\rho}\right)^{\alpha-\gamma-1} \tau^{\rho-1} \times \varphi_{r}\left(\int_{0}^{\tau} q\left(s, u(s),{ }^{C} \mathfrak{D}^{\gamma, \rho} u(s)\right) d s\right) d \tau \\
\quad-\frac{\rho^{2-\alpha+\gamma} t^{\rho(1-\gamma)}}{\Gamma(2-\gamma) \Gamma(\alpha-1)} \int_{0}^{1}\left(1-\tau^{\rho}\right)^{\alpha-2} \tau^{\rho-1} \varphi_{r}\left(\int_{0}^{\tau} q\left(s, u(s),{ }^{C} \mathfrak{D}^{\gamma, \rho} u(s)\right) d s\right) d \tau .
\end{aligned}
$$

The next result depends on the Banach fixed point theorem.

Theorem 10 Suppose that (H1)-(H3) are valid. If $\Lambda_{1}+\Lambda_{2}<1$, then the BoVaPr in (3) has a unique solution.

Proof We consider the set $B_{l}=\left\{u \in \mathbf{U}:\|u\|_{\mathbf{U}} \leq l\right\}$.

For each $t \in[0,1], u \in B_{l}$, we have the following estimation:

$$
\begin{aligned}
\left|\int_{0}^{\tau} q\left(s, u(s),{ }^{C} \mathfrak{D}^{\gamma, \rho} u(s)\right) d \tau\right| & \leq \int_{0}^{\tau}\left|q\left(s, x(s),{ }^{C} \mathfrak{D}^{\gamma, \rho} u(s)\right)-q(s, 0,0)+q(s, 0,0)\right| d s \\
& \leq \int_{0}^{\tau}\left(L_{q}\left(|u(s)|+\left|{ }^{C} \mathfrak{D}^{\gamma, \rho} u(s)\right|\right)+|q(s, 0,0)|\right) d s \\
& \leq \int_{0}^{1}\left(L_{q}\left(\|u\|+\left\|{ }^{C} \mathfrak{D}^{\gamma, \rho} u\right\|\right)+q^{*}\right) d s \\
& \leq L_{q} l+q^{*}=M .
\end{aligned}
$$

By Definition 5, we get $\varphi_{r}(M)=M^{r-1}, M>0$. 
We shall show that $\Psi B_{l} \subset B_{l}$. For each $t \in[0,1], x \in B_{l}$, and $r \geq 2$, we have

$$
\begin{aligned}
|\Psi u(t)| \leq & \frac{|\theta(u(0), u(1))|}{|\mu+1|}+\frac{|\mu||v(u(0), u(1))|}{\rho|\mu+1|}+\frac{|v(u(0), u(1))|}{\rho} t^{\rho} \\
& +\left|\frac{\rho^{1-\alpha}}{\Gamma(\alpha)} \int_{0}^{t}\left(t^{\rho}-\tau^{\rho}\right)^{\alpha-1} \tau^{\rho-1} \varphi_{r}\left(\int_{0}^{\tau} q\left(s, u(s),{ }^{C} \mathfrak{D}^{\gamma, \rho} u(s)\right) d s\right) d \tau\right| \\
& +\left|\frac{\rho^{2-\alpha} t^{\rho}}{\Gamma(\alpha-1)} \int_{0}^{1}\left(1-\tau^{\rho}\right)^{\alpha-2} \tau^{\rho-1} \varphi_{r}\left(\int_{0}^{\tau} q\left(s, u(s),{ }^{C} \mathfrak{D}^{\gamma, \rho} u(s)\right) d s\right) d \tau\right| \\
& +\left|\frac{\mu \rho^{1-\alpha}}{(\mu+1) \Gamma(\alpha)} \int_{0}^{1}\left(1-\tau^{\rho}\right)^{\alpha-1} \tau^{\rho-1} \varphi_{r}\left(\int_{0}^{\tau} q\left(s, u(s),{ }^{C} \mathfrak{D}^{\gamma, \rho} u(s)\right) d s\right) d \tau\right| \\
& +\left|\frac{\mu \rho^{2-\alpha}}{(\mu+1) \Gamma(\alpha-1)} \int_{0}^{1}\left(1-\tau^{\rho}\right)^{\alpha-2} \tau^{\rho-1} \varphi_{r}\left(\int_{0}^{\tau} q\left(s, u(s),{ }^{C} \mathfrak{D}^{\gamma, \rho} u(s)\right) d s\right) d \tau\right| \\
\leq & \frac{|\theta(u(0), u(1))|}{|\mu+1|}+\frac{|\mu||v(u(0), u(1))|}{\rho|\mu+1|}+\frac{|v(u(0), u(1))|}{\rho} t^{\rho} \\
& +\frac{M^{r-1} t^{\rho \alpha}}{\rho^{\alpha} \Gamma(\alpha+1)}+\frac{M^{r-1} t^{\rho}}{\rho^{\alpha-1} \Gamma(\alpha)}+\frac{M^{r-1}|\mu|}{\rho^{\alpha}|\mu+1| \Gamma(\alpha+1)}+\frac{M^{r-1}|\mu|}{\rho^{\alpha-1}|\mu+1| \Gamma(\alpha)} \\
\leq & \frac{M_{\theta}}{|\mu+1|}+\frac{|\mu| M_{v}}{\rho|\mu+1|}+\frac{M_{v}}{\rho}+\frac{(\alpha \rho+1) \mid(2|\mu|+1) M^{r-1}}{\rho^{\alpha}|\mu+1| \Gamma(\alpha+1)} .
\end{aligned}
$$

Moreover, we have

$$
\begin{aligned}
\left|{ }^{C} \mathfrak{D}^{\gamma, \rho} \Psi u(t)\right| \leq & \frac{|v(u(0), u(1))|}{\rho^{1-\gamma} \Gamma(2-\gamma)}+\frac{M^{r-1} \rho^{1-\alpha+\gamma}}{\Gamma(\alpha-\gamma)} \int_{0}^{t}\left(t^{\rho}-\tau^{\rho}\right)^{\alpha-\gamma-1} \tau^{\rho-1} d \tau \\
& +\frac{M^{r-1} \rho^{2-\alpha+\gamma} t^{\rho(1-\gamma)}}{\Gamma(2-\gamma) \Gamma(\alpha-1)} \int_{0}^{1}\left(1-\tau^{\rho}\right)^{\alpha-2} \tau^{\rho-1} d \tau \\
\leq & \frac{M_{v}}{\rho^{1-\gamma} \Gamma(2-\gamma)}+\frac{M^{r-1}}{\rho^{\alpha-\gamma} \Gamma(\alpha-\gamma+1)}+\frac{M^{r-1}}{\rho^{\alpha-\gamma-1} \Gamma(2-\gamma) \Gamma(\alpha)} .
\end{aligned}
$$

Thus, we get $\|\Psi u\|_{\mathrm{U}} \leq l$, which implies that $\Psi B_{l} \subset B_{l}$. Now, for each $t \in[0,1], u, m \in B_{l}$, and $r \geq 2$, we have

$$
\begin{aligned}
\mid \Psi u(t) & -\Psi m(t) \mid \\
\leq & \frac{\rho^{1-\alpha}}{\Gamma(\alpha)} \int_{0}^{t}\left(t^{\rho}-\tau^{\rho}\right)^{\alpha-1} \tau^{\rho-1} \\
& \times\left|\varphi_{r}\left(\int_{0}^{\tau} q\left(s, u(s),{ }^{C} \mathfrak{D}^{\gamma, \rho} u(s)\right) d s\right)-\varphi_{r}\left(\int_{0}^{\tau} q\left(s, m(s),{ }^{C} \mathfrak{D}^{\gamma, \rho} m(s)\right) d s\right)\right| d \tau \\
& +\frac{\rho^{2-\alpha} t^{\rho}}{\Gamma(\alpha-1)} \int_{0}^{1}\left(1-\tau^{\rho}\right)^{\alpha-2} \tau^{\rho-1} \\
& \times\left|\varphi_{r}\left(\int_{0}^{\tau} q\left(s, u(s),{ }^{C} \mathfrak{D}^{\gamma, \rho} u(s)\right) d s\right)-\varphi_{r}\left(\int_{0}^{\tau} q\left(s, m(s),{ }^{C} \mathfrak{D}^{\gamma, \rho} m(s)\right) d s\right)\right| d \tau \\
& +\frac{|\mu| \rho^{1-\alpha}}{|\mu+1| \Gamma(\alpha)} \int_{0}^{1}\left(1-\tau^{\rho}\right)^{\alpha-1} \tau^{\rho-1} \\
& \times\left|\varphi_{r}\left(\int_{0}^{\tau} q\left(s, u(s),{ }^{C} \mathfrak{D}^{\gamma, \rho} u(s)\right) d s\right)-\varphi_{r}\left(\int_{0}^{\tau} q\left(s, m(s),{ }^{C} \mathfrak{D}^{\gamma, \rho} m(s)\right) d s\right)\right| d \tau
\end{aligned}
$$




$$
\begin{aligned}
& +\frac{|\mu| \rho^{2-\alpha}}{|\mu+1| \Gamma(\alpha-1)} \int_{0}^{1}\left(1-\tau^{\rho}\right)^{\alpha-2} \tau^{\rho-1} \\
& \times\left|\varphi_{r}\left(\int_{0}^{\tau} q\left(s, u(s),{ }^{C} \mathfrak{D}^{\gamma, \rho} u(s)\right) d s\right)-\varphi_{r}\left(\int_{0}^{\tau} q\left(s, m(s),{ }^{C} \mathfrak{D}^{\gamma, \rho} m(s)\right) d s\right)\right| d \tau \\
& \leq \frac{(r-1) M^{r-2} L_{q} \rho^{1-\alpha}}{\Gamma(\alpha)} \int_{0}^{t}\left(t^{\rho}-\tau^{\rho}\right)^{\alpha-1} \tau^{\rho-1} \\
& \times \int_{0}^{\tau}\left(|u(s)-m(s)|+\left|{ }^{C} \mathfrak{D}^{\gamma, \rho} u(s)-{ }^{C} \mathfrak{D}^{\gamma, \rho} m(s)\right|\right) d s d \tau \\
& +\frac{(r-1) M^{r-2} L_{q} \rho^{2-\alpha} t^{\rho}}{\Gamma(\alpha-1)} \int_{0}^{1}\left(1-\tau^{\rho}\right)^{\alpha-2} \tau^{\rho-1} \\
& \times \int_{0}^{\tau}\left(|u(s)-m(s)|+\left|{ }^{C} \mathfrak{D}^{\gamma, \rho} u(s)-{ }^{C} \mathfrak{D}^{\gamma, \rho} m(s)\right|\right) d s d \tau \\
& +\frac{(r-1) M^{r-2} L_{q}|\mu| \rho^{1-\alpha}}{|\mu+1| \Gamma(\alpha)} \int_{0}^{1}\left(1-\tau^{\rho}\right)^{\alpha-1} \tau^{\rho-1} \\
& \times \int_{0}^{\tau}\left(|u(s)-m(s)|+\left|{ }^{C} \mathfrak{D}^{\gamma, \rho} u(s)-{ }^{C} \mathfrak{D}^{\gamma, \rho} m(s)\right|\right) d s d \tau \\
& +\frac{(r-1) M^{r-2} L_{q}|\mu| \rho^{2-\alpha}}{|\mu+1| \Gamma(\alpha-1)} \int_{0}^{1}\left(1-\tau^{\rho}\right)^{\alpha-2} \tau^{\rho-1} \\
& \times \int_{0}^{\tau}\left(|u(s)-m(s)|+\left|{ }^{C} \mathfrak{D}^{\gamma, \rho} u(s)-{ }^{C} \mathfrak{D}^{\gamma, \rho} m(s)\right|\right) d s d \tau \\
& \leq \frac{(r-1) M^{r-2} L_{q}}{\rho^{\alpha}}\left[\frac{\mathbf{B}\left(\alpha, \frac{1}{\rho}+1\right)}{\Gamma(\alpha)}+\frac{\rho \mathbf{B}\left(\alpha-1, \frac{1}{\rho}+1\right)}{\Gamma(\alpha-1)}\right. \\
& \left.+\frac{|\mu| \mathbf{B}\left(\alpha, \frac{1}{\rho}+1\right)}{|\mu+1| \Gamma(\alpha)}+\frac{\rho|\mu| \mathbf{B}\left(\alpha-1, \frac{1}{\rho}+1\right)}{|\mu+1| \Gamma(\alpha-1)}\right] \\
& \times\left(\|u-m\|+\left\|{ }^{C} \mathfrak{D}^{\gamma, \rho} u-{ }^{C} \mathfrak{D}^{\gamma, \rho} m\right\|\right)=\Lambda_{1}\|u-m\|_{\mathbf{U}} .
\end{aligned}
$$

Similarly, we have

$$
\begin{aligned}
\left\|{ }^{C} \mathfrak{D}^{\gamma, \rho} u(t)-{ }^{C} \mathfrak{D}^{\gamma, \rho} m(t)\right\| \leq & \frac{(r-1) M^{r-2} \rho^{1-\alpha+\gamma}}{\Gamma(\alpha-\gamma)} \int_{0}^{t}\left(t^{\rho}-\tau^{\rho}\right)^{\alpha-\gamma-1} \tau^{\rho-1} \\
& \times \int_{0}^{\tau}\left|q\left(s, u(s),{ }^{C} \mathfrak{D}^{\gamma, \rho} u(s)\right)-q\left(s, m(s),{ }^{C} \mathfrak{D}^{\gamma, \rho} m(s)\right)\right| d s d \tau \\
& +\frac{(r-1) M^{r-2} \rho^{2-\alpha+\gamma} t^{\rho(1-\gamma)}}{\Gamma(2-\gamma) \Gamma(\alpha-1)} \int_{0}^{1}\left(1-\tau^{\rho}\right)^{\alpha-2} \tau^{\rho-1} \\
& \times \int_{0}^{\tau}\left|q\left(s, u(s),{ }^{C} \mathfrak{D}^{\gamma, \rho} u(s)\right)-q\left(s, m(s),{ }^{C} \mathfrak{D}^{\gamma, \rho} m(s)\right)\right| d s d \tau \\
\leq & \frac{(r-1) M^{r-2} L_{q} \rho^{1-\alpha+\gamma}}{\Gamma(\alpha-\gamma)} \int_{0}^{t}\left(t^{\rho}-\tau^{\rho}\right)^{\alpha-\gamma-1} \tau^{\rho-1} \\
& \times \int_{0}^{\tau}\left(|u(s)-m(s)|+\left|{ }^{C} \mathfrak{D}^{\gamma, \rho} u(s)-{ }^{C} \mathfrak{D}^{\gamma, \rho} m(s)\right|\right) d s d \tau \\
& +\frac{(r-1) M^{r-2} L_{q} \rho^{2-\alpha+\gamma} t^{\rho(1-\gamma)}}{\Gamma(2-\gamma) \Gamma(\alpha-1)} \int_{0}^{1}\left(1-\tau^{\rho}\right)^{\alpha-2} \tau^{\rho-1} \\
& \times \int_{0}^{\tau}\left(|u(s)-m(s)|+\left|{ }^{C} \mathfrak{D}^{\gamma, \rho} u(s)-{ }^{C} \mathfrak{D}^{\gamma, \rho} m(s)\right|\right) d s d \tau
\end{aligned}
$$




$$
\begin{aligned}
\leq & \frac{(r-1) M^{r-2} L_{q}}{\rho^{\alpha-\gamma}}\left[\frac{\mathbf{B}\left(\alpha-\gamma, \frac{1}{\rho}+1\right)}{\Gamma(\alpha-\gamma)}+\frac{\rho \mathbf{B}\left(\alpha-1, \frac{1}{\rho}+1\right)}{\Gamma(2-\gamma) \Gamma(\alpha-1)}\right] \\
& \times\left(\|u-m\|+\left\|{ }^{C} \mathfrak{D}^{\gamma, \rho} u-{ }^{C} \mathfrak{D}^{\gamma, \rho} m\right\|\right) \\
= & \Lambda_{2}\|u-m\|_{\mathbf{U}} .
\end{aligned}
$$

Therefore, we get

$$
\|\Psi u-\Psi m\|_{\mathbf{U}} \leq\left(\Lambda_{1}+\Lambda_{2}\right)\|u-m\|_{\mathbf{U}}
$$

Since $\Lambda_{1}+\Lambda_{2}<1$ by the given assumption, then the operator $\Psi$ is a contraction. From the Banach fixed point theorem (Theorem 7), there exists a unique solution for the BoVaPr in (3). This completes the proof.

We now work on Schauder's fixed point by assuming the following:

(H4) There exist a function $\omega \in C\left([0,1], \mathbb{R}^{+}\right)$and a nondecreasing function $\Upsilon: \mathbb{R}^{+} \rightarrow \mathbb{R}^{+}, \Upsilon(0)=0$, such that:

$$
|q(t, u, m)| \leq \omega(t) \Upsilon(|u|+|m|), \quad \forall t \in[0,1], u, m \in \mathbb{R} .
$$

(H5) There exists a finite solution $l^{\prime}>0$ of the following inequality:

$$
\begin{aligned}
l^{\prime} \geq & \frac{M_{\theta}}{|\mu+1|}+\frac{|\mu| M_{\nu}}{\rho|\mu+1|}+\frac{M_{v}}{\rho}+\frac{M_{\nu}}{\rho^{1-\gamma} \Gamma(2-\gamma)}+\frac{\left(\|\omega\| \Upsilon\left(l^{\prime}\right)\right)^{r-1}}{\rho^{\alpha-\gamma} \Gamma(\alpha-\gamma+1)} \\
& +\frac{(2|\mu|+1)(\alpha \rho+1)\left(\|\omega\| \Upsilon\left(l^{\prime}\right)\right)^{r-1}}{\rho^{\alpha}|\mu+1| \Gamma(\alpha+1)}+\frac{\left(\|\omega\| \Upsilon\left(l^{\prime}\right)\right)^{r-1}}{\rho^{\alpha-\gamma-1} \Gamma(2-\gamma) \Gamma(\alpha)} .
\end{aligned}
$$

Theorem 11 Assume that (H2), (H4), and (H5) are satisfied. Then the BoVaPr in (3) has at least one solution.

Proof We transform the BoVaPr in (3) into a fixed point problem. Let us consider the operator $\Psi: \mathbf{U} \rightarrow \mathbf{U}$ defined by (H3), and let $B_{l^{\prime}}=\left\{u \in \mathbf{U}:\|u\|_{\mathbf{U}} \leq l^{\prime}\right\}$. Clearly, $B_{l^{\prime}}$ is a bounded closed convex set in $\mathbf{U}$. Next we shall show that $\Psi$ satisfies everything that is assumed in Theorem 8. Thus, $\Psi$ has a fixed point which is a solution of the BoVaPr in (3). The proof will be given step-by-step.

Step 1. $\Psi$ maps bounded sets into bounded sets in $\mathbf{U}$.

For each $t \in[0,1], u \in B_{l^{\prime}}$, we obtain

$$
\begin{aligned}
\left|\int_{0}^{\tau} q\left(s, u(s),{ }^{C} \mathfrak{D}^{\gamma, \rho} u(s)\right) d s\right| & \leq \int_{0}^{\tau}\left|q\left(s, u(s),{ }^{C} \mathfrak{D}^{\gamma, \rho} u(s)\right)\right| d s \\
& \leq \int_{0}^{\tau} \omega(s) \Upsilon\left(|u(s)|+\left|{ }^{C} \mathfrak{D}^{\gamma, \rho} u(s)\right|\right) d s \\
& \leq \int_{0}^{1}\|\omega\| \Upsilon\left(\|u\|+\left\|{ }^{C} \mathfrak{D}^{\gamma, \rho} u\right\|\right) d s \\
& \leq\|\omega\| \Upsilon\left(l^{\prime}\right) .
\end{aligned}
$$


By using Definition 5, we obtain the following estimate:

$$
\begin{aligned}
\left|\varphi_{r}\left(\int_{0}^{\tau} q\left(s, u(s),{ }^{C} \mathfrak{D}^{\gamma, \rho} u(s)\right) d s\right)\right| & =\left|\int_{0}^{\tau} q\left(s, u(s),{ }^{C} \mathfrak{D}^{\gamma, \rho} u(s)\right) d s\right|^{r-1} \\
& \leq\left(\|\omega\| \Upsilon\left(l^{\prime}\right)\right)^{r-1} .
\end{aligned}
$$

Therefore, we have

$$
\begin{aligned}
|\Psi u(t)| \leq & \frac{|\theta(u(0), u(1))|}{|\mu+1|}+\frac{|\mu||v(u(0), u(1))|}{\rho|\mu+1|}+\frac{|v(u(0), u(1))|}{\rho} \\
& +\frac{\left(\|\omega\| \Upsilon\left(l^{\prime}\right)\right)^{r-1} \rho^{1-\alpha}}{\Gamma(\alpha)} \int_{0}^{t}\left(t^{\rho}-\tau^{\rho}\right)^{\alpha-1} \tau^{\rho-1} d \tau \\
& +\frac{\left(\|\omega\| \Upsilon\left(l^{\prime}\right)\right)^{r-1} \rho^{2-\alpha} t^{\rho}}{\Gamma(\alpha-1)} \int_{0}^{1}\left(1-\tau^{\rho}\right)^{\alpha-2} \tau^{\rho-1} d \tau \\
& +\frac{|\mu|\left(\|\omega\| \Upsilon\left(l^{\prime}\right)\right)^{r-1} \rho^{1-\alpha}}{|\mu+1| \Gamma(\alpha)} \int_{0}^{1}\left(1-\tau^{\rho}\right)^{\alpha-1} \tau^{\rho-1} d \tau \\
& +\frac{|\mu|\left(\|\omega\| \Upsilon\left(l^{\prime}\right)\right)^{r-1} \rho^{2-\alpha}}{|\mu+1| \Gamma(\alpha-1)} \int_{0}^{1}\left(1-\tau^{\rho}\right)^{\alpha-2} \tau^{\rho-1} d \tau \\
\leq & \frac{M_{\theta}}{|\mu+1|}+\frac{|\mu| M_{v}}{\rho|\mu+1|}+\frac{M_{v}}{\rho}+\frac{(2|\mu|+1)(\alpha \rho+1)\left(\|\omega\| \Upsilon\left(l^{\prime}\right)\right)^{r-1}}{\rho^{\alpha}|\mu+1| \Gamma(\alpha+1)} .
\end{aligned}
$$

Moreover, we have

$$
\begin{aligned}
\left|\mathfrak{D}^{\gamma, \rho} \Psi u(t)\right| \leq & \frac{|v(u(0), u(1))|}{\rho^{1-\gamma} \Gamma(2-\gamma)}+\frac{\left(\|\omega\| \Upsilon\left(l^{\prime}\right)\right)^{r-1} \rho^{1-\alpha+\gamma}}{\Gamma(\alpha-\gamma)} \int_{0}^{t}\left(t^{\rho}-\tau^{\rho}\right)^{\alpha-\gamma-1} \tau^{\rho-1} d \tau \\
& +\frac{\left(\|\omega\| \Upsilon\left(l^{\prime}\right)\right)^{r-1} \rho^{2-\alpha+\gamma} t^{\rho(1-\gamma)}}{\Gamma(2-\gamma) \Gamma(\alpha-1)} \int_{0}^{1}\left(1-\tau^{\rho}\right)^{\alpha-2} \tau^{\rho-1} d \tau \\
\leq & \frac{M_{\nu}}{\rho^{1-\gamma} \Gamma(2-\gamma)}+\frac{\left(\|\omega\| \Upsilon\left(l^{\prime}\right)\right)^{r-1}}{\rho^{\alpha-\gamma} \Gamma(\alpha-\gamma+1)}+\frac{\left(\|\omega\| \Upsilon\left(l^{\prime}\right)\right)^{r-1}}{\rho^{\alpha-\gamma-1} \Gamma(2-\gamma) \Gamma(\alpha)} .
\end{aligned}
$$

Hence, we get

$$
\begin{aligned}
\|\Psi u\|_{\mathbf{U}} \leq & \frac{M_{\theta}}{|\mu+1|}+\frac{|\mu| M_{v}}{\rho|\mu+1|}+\frac{M_{v}}{\rho}+\frac{M_{v}}{\rho^{1-\gamma} \Gamma(2-\gamma)}+\frac{\left(\|\omega\| \Upsilon\left(l^{\prime}\right)\right)^{r-1}}{\rho^{\alpha-\gamma} \Gamma(\alpha-\gamma+1)} \\
& +\frac{(2|\mu|+1)(\alpha \rho+1)\left(\|\omega\| \Upsilon\left(l^{\prime}\right)\right)^{r-1}}{\rho^{\alpha}|\mu+1| \Gamma(\alpha+1)}+\frac{\left(\|\omega\| \Upsilon\left(l^{\prime}\right)\right)^{r-1}}{\rho^{\alpha-\gamma-1} \Gamma(2-\gamma) \Gamma(\alpha)} .
\end{aligned}
$$

In view of (H4), we deduce that $\Psi\left(B_{l^{\prime}}\right) \subset B_{l^{\prime}}$.

Step 2. $\Psi$ is continuous.

Assume that $\left\{u_{m}\right\}$ is a sequence where $u_{m} \rightarrow u$ in $\mathbf{U}$. We have

$$
\begin{aligned}
\left|\Psi u_{m}(t)-\Psi u(t)\right| \\
\leq \frac{\rho^{1-\alpha}}{\Gamma(\alpha)} \int_{0}^{t}\left(t^{\rho}-\tau^{\rho}\right)^{\alpha-1} \tau^{\rho-1} \\
\quad \times\left|\varphi_{r}\left(\int_{0}^{\tau} q\left(s, u_{n}(s),{ }^{C} \mathfrak{D}^{\gamma, \rho} u_{m}(s)\right) d s\right)-\varphi_{r}\left(\int_{0}^{\tau} q\left(s, u(s),{ }^{C} \mathfrak{D}^{\gamma, \rho} u(s)\right) d s\right)\right| d \tau
\end{aligned}
$$




$$
\begin{aligned}
& +\frac{\rho^{2-\alpha}}{\Gamma(\alpha-1)} \int_{0}^{1}\left(1-\tau^{\rho}\right)^{\alpha-2} \tau^{\rho-1} \\
& \times\left|\varphi_{r}\left(\int_{0}^{\tau} q\left(s, u_{m}(s),{ }^{C} \mathfrak{D}^{\gamma, \rho} u_{m}(s)\right) d s\right)-\varphi_{r}\left(\int_{0}^{\tau} q\left(s, u(s),{ }^{C} \mathfrak{D}^{\gamma, \rho} u(s)\right) d s\right)\right| d \tau \\
& +\frac{|\mu| \rho^{1-\alpha}}{|\mu+1| \Gamma(\alpha)} \int_{0}^{1}\left(1-\tau^{\rho}\right)^{\alpha-1} \tau^{\rho-1} \\
& \times\left|\varphi_{r}\left(\int_{0}^{\tau} q\left(s, u_{m}(s),{ }^{C} \mathfrak{D}^{\gamma, \rho} u_{m}(s)\right) d s\right)-\varphi_{r}\left(\int_{0}^{\tau} q\left(s, u(s),{ }^{C} \mathfrak{D}^{\gamma, \rho} u(s)\right) d s\right)\right| d \tau \\
& +\frac{|\mu| \rho^{2-\alpha}}{|\mu+1| \Gamma(\alpha-1)} \int_{0}^{1}\left(1-\tau^{\rho}\right)^{\alpha-2} \tau^{\rho-1} \\
& \times\left|\varphi_{r}\left(\int_{0}^{\tau} q\left(s, u_{m}(s),{ }^{C} \mathfrak{D}^{\gamma, \rho} u_{m}(s)\right) d s\right)-\varphi_{r}\left(\int_{0}^{\tau} q\left(s, u(s),{ }^{C} \mathfrak{D}^{\gamma, \rho} u(s)\right) d s\right)\right| d \tau .
\end{aligned}
$$

Then, by the dominated convergence theorem, for all $t \in[0,1]$, we have $\mid \Psi u_{m}(t)-$ $\Psi u(t) \mid \rightarrow 0$. Further, we have

$$
\begin{aligned}
\left|{ }^{C} \mathfrak{D}^{\gamma, \rho} u_{m}(t)-{ }^{C} \mathfrak{D}^{\gamma, \rho} u(t)\right| & \\
\leq & \frac{\rho^{1-\alpha+\gamma}}{\Gamma(\alpha-\gamma)} \int_{0}^{t}\left(t^{\rho}-\tau^{\rho}\right)^{\alpha-\gamma-1} \tau^{\rho-1} \\
& \times\left|\varphi_{r}\left(\int_{0}^{\tau} q\left(s, u_{m}(s),{ }^{C} \mathfrak{D}^{\gamma, \rho} u_{m}(s)\right) d s\right)-\varphi_{r}\left(\int_{0}^{\tau} q\left(s, u(s),{ }^{C} \mathfrak{D}^{\gamma, \rho} u(s)\right) d s\right)\right| d \tau \\
& +\frac{\rho^{2-\alpha+\gamma}}{\Gamma(2-\gamma) \Gamma(\alpha-1)} \int_{0}^{1}\left(1-\tau^{\rho}\right)^{\alpha-2} \tau^{\rho-1} \\
& \times\left|\varphi_{r}\left(\int_{0}^{\tau} q\left(s, u_{m}(s),{ }^{C} \mathfrak{D}^{\gamma, \rho} u_{m}(s)\right) d s\right)-\varphi_{r}\left(\int_{0}^{\tau} q\left(s, u(s),{ }^{C} \mathfrak{D}^{\gamma, \rho} u(s)\right) d s\right)\right| d \tau .
\end{aligned}
$$

In view of the dominated convergence theorem, we deduce that $\mid{ }^{C} \mathfrak{D}^{\gamma, \rho} u_{m}(t)-$ ${ }^{C} \mathfrak{D}^{\gamma, \rho} u(t) \mid \rightarrow 0$. Hence, we conclude that $\left\|\Psi u_{m}-\Psi u\right\|_{\mathbf{U}} \rightarrow 0$ in $\mathbf{U}$. Hence, $\Psi$ is continuous.

Step 3. $\left\{(\Psi u), u \in B_{l^{\prime}}\right\}$ and $\left\{{ }^{C} \mathfrak{D}^{\gamma, \rho}(\Psi u), u \in B_{l^{\prime}}\right\}$ are equicontinuous.

For $t_{1}<t_{2}$ with $t_{1}, t_{2} \in[0,1]$, we have

$$
\begin{aligned}
&\left|(\Psi u)\left(t_{2}\right)-(\Psi u)\left(t_{1}\right)\right| \\
& \leq\left|\frac{v(u(0), u(1))}{\rho}\left(t_{2}^{\rho}-t_{1}^{\rho}\right)\right| \\
& \quad+\left|\frac{\rho^{1-\alpha}}{\Gamma(\alpha)} \int_{t_{1}}^{t_{2}}\left(t_{2}^{\rho}-\tau^{\rho}\right)^{\alpha-1} \tau^{\rho-1} \varphi_{r}\left(\int_{0}^{\tau} q\left(s, u(s),{ }^{C} \mathfrak{D}^{\gamma, \rho} u(s)\right) d s\right) d \tau\right| \\
& \quad+\mid \frac{\rho^{1-\alpha}}{\Gamma(\alpha)} \int_{0}^{t_{1}}\left[\left(t_{2}^{\rho}-\tau^{\rho}\right)^{\alpha-1}-\left(t_{1}^{\rho}-\tau^{\rho}\right)^{\alpha-1}\right] \tau^{\rho-1} \varphi_{r} \\
& \quad \times\left(\int_{0}^{\tau} q\left(s, u(s),{ }^{C} \mathfrak{D}^{\gamma, \rho} u(s)\right) d s\right) \mid d \tau \\
& \quad+\left|\frac{\rho^{2-\alpha}\left(t_{2}^{\rho}-t_{1}^{\rho}\right)}{\Gamma(\alpha-1)} \int_{0}^{1}\left(1-\tau^{\rho}\right)^{\alpha-2} \tau^{\rho-1} \varphi_{r}\left(\int_{0}^{\tau} q\left(s, u(s),{ }^{C} \mathfrak{D}^{\gamma, \rho} u(s)\right) d s\right) d \tau\right| \\
& \leq \frac{M_{v}}{\rho}\left(t_{2}^{\rho}-t_{1}^{\rho}\right)+\frac{\left(\|\omega\| \Upsilon\left(l^{\prime}\right)\right)^{r-1} \rho^{1-\alpha}}{\Gamma(\alpha)} \int_{t_{1}}^{t_{2}}\left(t_{2}^{\rho}-\tau^{\rho}\right)^{\alpha-1} \tau^{\rho-1} d \tau
\end{aligned}
$$




$$
\begin{aligned}
& +\frac{\left(\|\omega\| \Upsilon\left(l^{\prime}\right)\right)^{r-1} \rho^{1-\alpha}}{\Gamma(\alpha)} \int_{0}^{t_{1}}\left|\left(t_{2}^{\rho}-\tau^{\rho}\right)^{\alpha-1}-\left(t_{1}^{\rho}-\tau^{\rho}\right)^{\alpha-1}\right| \tau^{\rho-1} d \tau \\
& +\frac{\left(\|\omega\| \Upsilon\left(l^{\prime}\right)\right)^{r-1} \rho^{1-\alpha}\left(t_{2}^{\rho}-t_{1}^{\rho}\right)}{\Gamma(\alpha-1)} \int_{0}^{1}\left(1-\tau^{\rho}\right)^{\alpha-2} \tau^{\rho-1} d \tau \\
& \leq \frac{M_{v}}{\rho}\left(t_{2}^{\rho}-t_{1}^{\rho}\right)+\frac{\left(\|\omega\| \Upsilon\left(l^{\prime}\right)\right)^{r-1}}{\rho^{\alpha} \Gamma(\alpha+1)}\left[2\left(t_{2}^{\rho}-t_{1}^{\rho}\right)^{\alpha}+t_{2}^{\rho \alpha}-t_{1}^{\rho \alpha}\right] \\
& +\frac{\left(\|\omega\| \Upsilon\left(l^{\prime}\right)\right)^{r-1}}{\rho^{\alpha} \Gamma(\alpha)}\left(t_{2}^{\rho}-t_{1}^{\rho}\right) .
\end{aligned}
$$

Thus, $\left|(\Psi u)\left(t_{2}\right)-(\Psi u)\left(t_{1}\right)\right| \rightarrow 0$ as $t_{2} \rightarrow t_{1}$. In addition, we have the following:

$$
\begin{aligned}
\left|{ }^{C} \mathfrak{D}^{\gamma, \rho}(\Psi u)\left(t_{2}\right)-{ }^{C} \mathfrak{D}^{\gamma, \rho}(\Psi u)\left(t_{1}\right)\right| & \\
\leq & \frac{M_{v}}{\rho^{1-\gamma} \Gamma(2-\gamma)}\left(t_{2}^{\rho(1-\gamma)}-t_{1}^{\rho(1-\gamma)}\right) \\
& +\frac{\left(\|\omega\| \Upsilon\left(l^{\prime}\right)\right)^{r-1} \rho^{1-\alpha+\gamma}}{\Gamma(\alpha-\gamma)} \int_{t_{1}}^{t_{2}}\left(t_{2}^{\rho}-\tau^{\rho}\right)^{\alpha-\gamma-1} \tau^{\rho-1} d \tau \\
& +\frac{\left(\|\omega\| \Upsilon\left(l^{\prime}\right)\right)^{r-1} \rho^{1-\alpha+\gamma}}{\Gamma(\alpha-\gamma)} \int_{0}^{t_{1}}\left|\left(t_{2}^{\rho}-\tau^{\rho}\right)^{\alpha-\gamma-1}-\left(t_{1}^{\rho}-\tau^{\rho}\right)^{\alpha-\gamma-1}\right| \tau^{\rho-1} d \tau \\
& +\frac{\left(\|\omega\| \Upsilon\left(l^{\prime}\right)\right)^{r-1} \rho^{2-\alpha+\gamma}\left(t_{2}^{\rho(1-\gamma)}-t_{1}^{\rho(1-\gamma)}\right)}{\Gamma(2-\gamma) \Gamma(\alpha-1)} \int_{0}^{1}\left(1-\tau^{\rho}\right)^{\alpha-2} \tau^{\rho-1} d \tau \\
\leq & \frac{M_{v}}{\rho^{1-\gamma} \Gamma(2-\gamma)}\left(t_{2}^{\rho(1-\gamma)}-t_{1}^{\rho(1-\gamma)}\right) \\
& +\frac{\left(\|\omega\| \Upsilon\left(l^{\prime}\right)\right)^{r-1}}{\rho^{\alpha-\gamma} \Gamma(\alpha-\gamma+1)}\left[2\left(t_{2}^{\rho}-t_{1}^{\rho}\right)^{\alpha-\gamma}+t_{2}^{\rho(\alpha-\gamma)}-t_{1}^{\rho(\alpha-\gamma)}\right] \\
& +\frac{\left(\|\omega\| \Upsilon\left(l^{\prime}\right)\right)^{r-1}}{\rho^{\alpha-\gamma-1} \Gamma(2-\gamma) \Gamma(\alpha)}\left(t_{2}^{\rho(1-\gamma)}-t_{1}^{\rho(1-\gamma)}\right) .
\end{aligned}
$$

Thus, $\left|{ }^{C} \mathfrak{D}^{\gamma, \rho}(\Psi u)\left(t_{2}\right)-{ }^{C} \mathfrak{D}^{\gamma, \rho}(\Psi u)\left(t_{1}\right)\right| \rightarrow 0$ as $t_{2} \rightarrow t_{1}$. Hence, $\left\{(\Psi u), u \in B_{l^{\prime}}\right\}$ and $\left\{{ }^{C} \mathfrak{D}^{\gamma, \rho}(\Psi u), u \in B_{l^{\prime}}\right\}$ are equicontinuous and relatively compact according to the ArzelàAscoli theorem. Thus, $\Psi\left(B_{l^{\prime}}\right)$ is a relatively compact subset of $\mathbf{U}$, and the operator $\Psi$ is compact. From Schauder's fixed point (see Theorem 8 ), $\Psi$ has a fixed point $x$ which is a solution of the BoVaPr in (3). The proof is completed.

\section{Application}

By choosing particular parameters, the following example is provided in this section to apply and validate all obtained results from the previous sections.

We construct an example with specific parameters to expose the applicability of the proposed theoretical results.

Example 1 Consider the following BoVaPr:

$$
\left\{\begin{array}{l}
\frac{d}{d t}\left(\varphi_{2}\left({ }^{C} \mathfrak{D}^{\frac{3}{2}, 2} u(t)\right)\right)=\frac{\arctan (t)}{1+\frac{1}{5}|u(t)|+\frac{1}{5}\left|{ }^{C} \mathfrak{D}^{\frac{1}{2}, 2} u(t)\right|} \quad(t \in[0,1]), \\
u(0)+u(1)=\sin u(0) \cos u(1), \quad u^{\prime}(1)=\cos u(0) \sin u(1) .
\end{array}\right.
$$


Here, $\alpha=\frac{3}{2}, \gamma=\frac{1}{2}, \rho=2, p=q=2, \mu=M_{v}=M_{\theta}=1$, and $q$ is expressed as

$$
q\left(t, u(t),{ }^{C} \mathfrak{D}^{\gamma, \rho} u(t)\right)=\frac{\arctan (t)}{1+\frac{1}{5}|u(t)|+\left.\frac{1}{5}\right|^{C} \mathfrak{D}^{\frac{1}{2}, 2} u(t) \mid} \quad(t \in[0,1]) .
$$

Clearly, $q:[0,1] \times \mathbb{R} \times \mathbb{R} \rightarrow \mathbb{R}$ is a continuous function, and we have

$$
\begin{aligned}
\left|q\left(t, u_{1}, m_{1}\right)-q\left(t, u_{2}, m_{2}\right)\right| & =\left|\frac{\arctan (t)}{1+\frac{1}{5}\left|u_{1}\right|+\frac{1}{5}\left|m_{1}\right|}-\frac{\arctan (t)}{1+\frac{1}{5}\left|u_{2}\right|+\frac{1}{5}\left|m_{2}\right|}\right| \\
& \leq \frac{\pi}{20}\left(\left|u_{1}-u_{2}\right|+\left|m_{1}-m_{2}\right|\right)
\end{aligned}
$$

Thus, assumption (H1) is satisfied with $L_{q}=\frac{\pi}{20}$. Also, we have

$$
q^{*}=\sup _{t \in[0,1]}|q(t, 0,0)|=\frac{\pi}{4}
$$

and

$$
\Lambda_{1}+\Lambda_{2}=0.2091745071+0.235619449=0.4447939561<1 .
$$

As a result, all of the assumptions we made in Theorem 10 are satisfied. Hence, the BoVaPr in (13) has a unique solution on $[0,1]$.

Unfortunately, the functions $\omega(t)=\arctan (t)$ and $\Upsilon(s)=\frac{1}{1+\frac{1}{5} s}$ do not satisfy assumption (H4). Alternatively, the function

$$
q(t, u, m)=\arctan (t) \frac{|u(t)|+|m(t)|}{1+|u(t)|+|m(t)|} \quad(t \in[0,1]),
$$

with $\omega(t)=\arctan (t)$ and $\Upsilon(s)=\frac{s}{s+1}$ satisfies condition (H4). Moreover, estimate (12) can be simplified as

$$
l^{\prime} \geq 2.0479+\frac{2.6454 l^{\prime}}{l^{\prime}+1}
$$

Hence, any real number $l^{\prime}>4.2$ satisfies assumption (H5). Therefore, everything assumed in Theorem 11 is satisfied, and then there exists a solution of problem (13).

\section{Conclusion}

The existence and uniqueness of solutions for the proposed $p$-Laplacian nonperiodic boundary value problem in the sense of generalized Caputo fractional derivative have been successfully investigated in this research paper using the Banach and Schauder fixed point theorems. All the obtained results are supported by an applicable example to apply and validate them. Therefore, this research study sheds the light on this interesting topic of research and motivates all other researchers to work on further investigation of $p$-Laplacian nonperiodic boundary value problem defined in other fractional derivatives. 
Funding

Not applicable.

\section{Availability of data and materials}

Data sharing not applicable to this article as no datasets were generated or analyzed during the current study.

\section{Ethics approval and consent to participate}

Not applicable.

\section{Competing interests}

The authors declare that they have no competing interests.

\section{Consent for publication}

Not applicable.

\section{Authors' contributions}

The authors declare that the study was realized in collaboration with equal responsibility. All authors read and approved the final manuscript.

\section{Authors' information}

(M.M. Matar:mohammed_mattar@hotmail.com; M.I. Abbas: miabbas77@gmail.com; J. Alzabut: jalzabut@psu.edu.sa; M.K.A. Kaabar: mohammedkaabar@gmail.com; S. Etemad: sina.etemad@gmail.com)

\section{Author details}

'Department of Mathematics, Al-Azhar University-Gaza, Gaza, Palestine. ${ }^{2}$ Department of Mathematics and Computer Science, Faculty of Science, Alexandria University, Alexandria 21511, Egypt. ${ }^{3}$ Department of Mathematics and General Sciences, Prince Sultan University, Riyadh 11586, Saudi Arabia. ${ }^{4}$ Department of Mathematics and Statistics, Washington State University, Pullman, WA, USA. ${ }^{5}$ Department of Mathematics, Azarbaijan Shahid Madani University, Tabriz, Iran. ${ }^{6}$ Institute of Research and Development, Duy Tan University, Da Nang 550000, Vietnam. ${ }^{7}$ Faculty of Natural Sciences, Duy Tan University, Da Nang 550000, Vietnam. ${ }^{8}$ Department of Medical Research, China Medical University Hospital, China Medical University, Taichung, Taiwan.

\section{Publisher's Note}

Springer Nature remains neutral with regard to jurisdictional claims in published maps and institutional affiliations.

Received: 1 September 2020 Accepted: 11 January 2021 Published online: 22 January 2021

\section{References}

1. Alzabut, J., Abdeljawad, T., Alrabaiah, H.: Oscillation criteria for forced and damped nabla fractional difference equations. J. Comput. Anal. Appl. 24(8), 1387-1394 (2018)

2. Kaabar, M.: Novel methods for solving the conformable wave equation. J. New Theory 31, $56-85$ (2019)

3. Kilbas, A.A., Srivastava, H.M., Trujillo, J.J.: Theory and Applications of Fractional Differential Equations. Elsevier, Amsterdam (2006)

4. Samko, S.G., Kilbas, A.A., Marichev, O.I.: Fractional Integrals and Derivatives: Theory and Applications. Gordon \& Breach, Yverdon (1993)

5. Martinez, F., Martinez, I., Kaabar, M.K.A., Ortiz-Munuera, R., Paredes, S.: Existence of mild solution for hybrid differential equations with arbitrary fractional order. IAENG Int. J. Appl. Math. 50(3), 18 (2020)

6. Baleanu, D., Etemad, S., Rezapour, S.: On a fractional hybrid integro-differential equation with mixed hybrid integral boundary value conditions by using three operators. Alex. Eng. J. (2020). https://doi.org/10.1016/j.aej.2020.04.053

7. Baleanu, D., Rezapour, S., Mohammadi, H.: Some existence results on nonlinear fractional differential equations. Philos. Trans. R. Soc. A 371, 20120144 (2013). https://doi.org/10.1098/rsta.2012.0144

8. Agarwal, R.P., Baleanu, D., Hedayati, V., Rezapour, S.: Two fractional derivative inclusion problems via integral boundary conditions. Appl. Math. Comput. 257, 205-212 (2015). https://doi.org/10.1016/j.amc.2014.10.082

9. Baleanu, D., Hedayati, V., Rezapour, S.: On two fractional differential inclusions. SpringerPlus 5, 882 (2016). https://doi.org/10.1186/s40064-016-2564-z

10. Etemad, S., Rezapour, S., Samei, M.E.: On a fractional Caputo-Hadamard inclusion problem with sum boundary value conditions by using approximate endpoint property. Math. Model. Appl. Sci. 43(17), 9719-9734 (2020). https://doi.org/10.1002/mma.6644

11. Rezapour, S., Samei, M.E.: On the existence of solutions for a multi-singular pointwise defined fractional q-integro-differential equation. Bound. Value Probl. 2020, 38 (2020). https://doi.org/10.1186/s13661-020-01342-3

12. Aydogan, S.M., Baleanu, D., Aguilar, J.F.G., Rezapour, S.: Approximate endpoint solutions for a class of fractional q-differential inclusions. Fractals 28(8), 2040029 (2020). https://doi.org/10.1142/S0218348X20400290

13. Jarad, F., Abdeljawad, T., Alzabut, J.: On the generalized fractional derivatives and their Caputo modification. Eur. Phys J. Spec. Top. 226, 3457-3471 (2017). https://doi.org/10.1140/epjst/e2018-00021-7

14. Seemab, A., Rehman, M.U., Alzabut, J., Hamdi, A.: On the existence of positive solutions for generalized fractional boundary value problems. Bound. Value Probl. 2019, 186 (2019). https://doi.org/10.1186/s13661-019-01300-8

15. Abbas, M.l.: Ulam stability of fractional impulsive differential equations with Riemann-Liouville integral boundary conditions. J. Contemp. Math. Anal. 50(5), 209-219 (2015)

16. Abbas, M.I.: Existence and uniqueness of solution for a boundary value problem of fractional order involving two Caputo's fractional derivatives. Adv. Differ. Equ. 2015, 252 (2015). https://doi.org/10.1186/s13662-015-0581-9 
17. Ahmad, B., Etemad, S., Ettefagh, M., Rezapour, S.: On the existence of solutions for fractional q-difference inclusions with q-antiperiodic boundary conditions. Bull. Math. Soc. Sci. Math. Roum. 59(107)(2), 119-134 (2016)

18. Ahmad, B., Matar, M.M., Al-Salmy, Q.M.: Existence of solutions and Ulam stability for Caputo type sequential fractional differential equations of order $\alpha \in(2,3)$. Int. J. Anal. Appl. 15, 86-101 (2017)

19. Ahmad, B., Matar, M.M., Ntouyas, S.K.: On general fractional differential inclusions with nonlocal integral boundary conditions. Differ. Equ. Dyn. Syst. 28, 241-254 (2020). https://doi.org/10.1007/s12591-016-0319-5

20. Aydogan, M., Baleanu, D., Mousalou, A., Rezapour, S.: On high order fractional integro-differential equations including the Caputo-Fabrizio derivative. Bound. Value Probl. 2018, 90 (2018). https://doi.org/10.1186/s13661-018-1008-9

21. Baleanu, D., Etemad, S., Rezapour, S.: A hybrid Caputo fractional modeling for thermostat with hybrid boundary value conditions. Bound. Value Probl. 2020, 64 (2020). https://doi.org/10.1186/s13661-020-01361-0

22. Baleanu, D., Mousalou, A., Rezapour, S.: On the existence of solutions for some infinite coefficient-symmetric Caputo-Fabrizio fractional integro-differential equations. Bound. Value Probl. 2017(1), 145 (2017) https://doi.org/10.1186/s13661-017-0867-9

23. Baleanu, D., Rezapour, S., Etemad, S., Alsaedi, A.: On a time-fractional integro-differential equation via three-point boundary value conditions. Math. Probl. Eng. 2015, 785738 (2015). https://doi.org/10.1155/2015/785738

24. Berhail, A., Tabouche, N., Matar, M.M., Alzabut, J.: On nonlocal integral and derivative boundary value problem of nonlinear Hadamard Langevin equation with three different fractional orders. Bol. Soc. Mat. Mex. 26, 303-318 (2020). https://doi.org/10.1007/s40590-019-00257-z

25. Etemad, S., Ntouyas, S.K., Ahmad, B.: Existence theory for a fractional $q$-integro-difference equation with $q$-integral boundary conditions of different orders. Mathematics 7, 659 (2019). https://doi.org/10.3390/math7080659

26. Mahmudov, N.I., Matar, M.M.: Existence of mild solution for hybrid differential equations with arbitrary fractional order. TWMS J. Pure Appl. Math. 8(2), 160-169 (2017)

27. Matar, M.M.: Existence of solution involving Genocchi numbers for nonlocal anti-periodic boundary value problem of arbitrary fractional order. Rev. R. Acad. Cienc. Exactas Fís. Nat., Ser. A Mat. 112, 945-956 (2017). https://doi.org/10.1007/s13398-017-0403-x

28. Matar, M.M.: Solution of sequential Hadamard fractional differential equations by variation of parameter technique. Abstr. Appl. Anal. 2018, 9605353 (2018). https://doi.org/10.1155/2018/9605353

29. Matar, M.M., Abu Skhail, S.E.: On stability of nonautonomous perturbed semilinear fractional differential systems of order $\alpha \in(1,2)$. J. Math. 2018, 1723481 (2018). https://doi.org/10.1155/2018/1723481

30. Matar, M.M., Al-Salmy, O.M.: Existence and uniqueness of solution for conformable sequential differential equations. J. Al Azhar Univ.-Gaza Nat. Sci. 19, 41-56 (2017)

31. Matar, M.M.: Existence of integral and anti-periodic boundary valued problem of fractional order $0<\alpha \leq 3$. Bull. Malays. Math. Sci. Soc. 40(3), 959-973 (2017). https://doi.org/10.1007/s40840-016-0332-4

32. Matar, M.M., Trujillo, J.J.: Existence of local solutions for differential equations with arbitrary fractional order. Arab. J. Math. 5, 215-224 (2016). https://doi.org/10.1007/s40065-015-0139-4

33. Matar, M.M., Abu Skhail, S.E., Alzabut, J.: On solvability of nonlinear fractional differential systems involving nonlocal initial conditions. Math. Methods Appl. Sci. (2019). https://doi.org/10.1002/mma.5910

34. Ntouyas, S.K., Etemad, S.: On the existence of solutions for fractional differential inclusions with sum and integral boundary conditions. Appl. Math. Comput. 266, 235-243 (2015). https://doi.org/10.1016/j.amc.2015.05.036

35. Zhou, H., Alzabut, J., Yang, L.: On fractional Langevin differential equations with anti-periodic boundary conditions. Eur. Phys. J. Spec. Top. 226, 3577-3590 (2017). https://doi.org/10.1140/epjst/e2018-00082-0

36. Liu, X., Jia, M.: The method of lower and upper solutions for the general boundary value problems of fractional differential equations with p-Laplacian. Adv. Differ. Equ. 2018, 28 (2018). https://doi.org/10.1186/s13662-017-1446-1

37. Jafari, H., Baleanu, D., Khan, H.: Existence criterion for the solutions of fractional order $p$-Laplacian boundary value problems. Bound. Value Probl. 2015, 164 (2015). https://doi.org/10.1186/s13661-015-0425-2

38. Liu, X., Jia, M., Xiang, X.: On the solvability of a fractional differential equation model involving the $p$-Laplacian operator. Comput. Math. Appl. 64, 3267-3275 (2012). https://doi.org/10.1016/j.camwa.2012.03.001

39. Liu, Z., Lu, L.: On the solvability of a fractional differential equation model involving the $p$-Laplacian operator. Electron J. Qual. Theory Differ. Equ. 2012, 70 (2012). https://doi.org/10.14232/ejqtde.2012.1.70

40. Mahmudov, N.I., Unul, S.: On the solvability of a fractional differential equation model involving the $p$-Laplacian operator. Bound. Value Probl. 2015, 99 (2015). https://doi.org/10.1186/s13661-015-0358-9

41. Tang, X., Yan, C., Liu, Q.: Existence of solutions of two-point boundary value problems for fractional $p$-Laplace differential equations at resonance. J. Appl. Math. Comput. 41, 119-131 (2013). https://doi.org/10.1007/s12190-012-0598-0

42. Almeida, R., Malinowska, A.B., Odzijewicz, T.: Fractional differential equations with dependence on the Caputo-Katugampola derivative. J. Comput. Nonlinear Dyn. 11(6), 061017 (2016)

43. Cao, L., Kong, H., Zeng, S.D.: Maximum principles for time-fractional Caputo-Katugampola diffusion equations. J. Nonlinear Sci. Appl. 10, 2257-2267 (2017). https://doi.org/10.22436/jnsa.010.04.75

44. Zeng, S., Baleanu, D., Bai, Y., Wu, G.: Fractional differential equations of Caputo-Katugampola type and numerical solutions. Appl. Math. Comput. 315, 549-554 (2017). https://doi.org/10.1016/j.amc.2017.07.003

45. Hoa, N.V., Vu, H., Duc, T.M.: Fuzzy fractional differential equations under Caputo-Katugampola fractional derivative approach. Fuzzy Sets Syst. 375, 70-99 (2019). https://doi.org/10.1016/j.fss.2018.08.001

46. Baleanu, D., Aydogan, S.M., Mohammadi, H., Rezapour, S.: On modelling of epidemic childhood diseases with the Caputo-Fabrizio derivative by using the Laplace Adomian decomposition method. Alex. Eng. J. (2020). https://doi.org/10.1016/j.aej.2020.05.007

47. Tuan, N.H., Mohammadi, H., Rezapour, S.: A mathematical model for COVID-19 transmission by using the Caputo fractional derivative. Chaos Solitons Fractals 140, 110107 (2020). https://doi.org/10.1016/j.chaos.2020.110107

48. Baleanu, D., Jajarmi, A., Mohammadi, H., Rezapour, S.: Analysis of the human liver model with Caputo-Fabrizio fractional derivative. Chaos Solitons Fractals 134, 109705 (2020)

49. Jarad, F., Abdeljawad, T., Baleanu, D.: On the generalized fractional derivatives and their Caputo modification. J. Nonlinear Sci. Appl. 10, 2607-2619 (2017). https://doi.org/10.22436/jnsa.010.05.27

50. Smart, D.R.: Fixed Point Theorems. Cambridge University Press, New York (1980) 